ISSN: 2379-2922

Volume 5, Issue 1, 16 Pages

\title{
Association between Insomnia, Demographic Characteristics and Self - Esteem in Nursing Personnel in Primary Care and in Emergency - Intensive Care Units on the Island of Crete
}

\section{Maria Maniou $^{1 *}$, Sofia Zyga ${ }^{2}$, Spyros Vliamos ${ }^{3}$, Panagiotis Prezerakos ${ }^{4}$, Katerina Flora ${ }^{5}$} Andreas Pavlakis ${ }^{6}$

${ }^{1 *}$ Nurse - Computer Engineer, MSc, PhD(c), Organization of Social Solidarity and Education. Municipality of Archanes - Asterousia,

Faculty of Nursing - Technological Educational Institute of Crete, Iraklion, Greece.

${ }^{2}$ Associate Professor, MSc, PhD, Faculty of Nursing, University of Peloponnese, Sparta, Greece.

${ }^{3}$ Professor, Neapolis University, Pafos, Cyprus.

${ }^{4}$ Associate Professor, MSc, PhD, Faculty of Nursing, University of Peloponnese, Sparta, Greece.

${ }^{5}$ Lecturer, MSc, PhD, Neapolis University, Pafos, Cyprus.

${ }^{6}$ Associate Professor, MSc, PhD, Neapolis University, Pafos, Cyprus.

*mmaniou@hotmail.com

\section{Abstract}

Background: There is evidence that self - esteem is important for health. Especially, self - esteem is associated with better health in those with chronic conditions or disability.

Purpose: The objective of this study was to examine, in a comprehensive way: (a) the association between insomnia and self-esteem, (b) the association between insomnia and marital status in nursing personnel in the island of Crete, Greece.

Method: An investigation was carried out in the area of Crete between August 2017 and January 2018 in fourteen Health Centers, two Primary National Health Networks, four Emergency Department, eleven Intensive Care Units. The study involved 213 nurses. The Hamilton Anxiety Scale (HAM-A) and the Culture-free Selfesteem Inventories had been used.

Results: Prevalence of insomnia was 49.3\% (from mild to very severe symptoms). Multivariate analyses showed that self-esteem was significantly associated with insomnia symptoms $(p<0,002)$. Multivariate models also showed that insomnia-type symptoms were associated with marital status $(p<0,026)$.

Conclusions: In a large population of nursing personnel insomnia symptoms are prevalent and associated with self-esteem and marital status. Future studies should assess whether the strengthening of self- esteem improves the sleep problems in nursing personnel.

Keywords: Insomnia symptoms, Self-esteem, Marital status, Nursing Personnel

\section{INTRODUCTION}

Studies reports that the rate of insomnia in the united states, valued at $10 \%$ to $40 \%$, and continue to raise these percentages, researchers try to understand and treat sleep disorders.1,2

Chronic insomnia is associated with psychiatric and physical conditions. Although it is a symptom of depression, insomnia is also a forerunner of depression and is associated with a substantial increase in the relative risk of major depression. Chronic insomnia is associated with deteriorated mood, subjective functioning, quality of 
Association between Insomnia, Demographic Characteristics and Self - Esteem in Nursing Personnel in Primary Care and in Emergency - Intensive Care Units on the Island of Crete

life and increased daytime sleepiness. Chronic insomnia is a common problem, often associated with negative waking mood or function [3]. Anxiety disorders and comorbid mood are associated with high rates of severe insomnia, which were independently associated with functional impairment [4]. Insomnia is associated with anxiety and disorders mood, and longitudinal evidence shows that insomnia predates the onset of mental illness [5].

Further, in a study, Lemola et al.[6] demonstrated that short sleep (sleeping less than 6 hours) was negatively related to self - esteem. Also, the same study showed that long sleep (sleeping more than 9 hours) was negatively related to self-esteem. Moreover, the relation of long sleep with lower self-esteem was statistically significant when adjusting insomnia symptoms.

According Gregory et al., family influences may be important in the association between insomnia [7]. Marital status is considered to be of importance among the sociodemographic determinants of insomnia. Furthermore, although in univariate analysis the odds for insomnia was lower for single persons but higher for divorced or widowed persons compared to married subjects. This association was not statically significant in multivariate analysis. Therefore, marital status did not subscribe to the occurrence of insomnia contrary to what is generally reported in the literature $[8,9]$. In another study, it was not found significant association between marital status and insomnia in males. However, divorced/widowed females were more conquerable to insomnia (age-adjusted OR 1.78; 95\% CI 1.20-2.63) [10].

The gender differences marriages have been suggested as disadvantageous to females because of genderspecific demand and burden as well as restricted roles available to females in the marriage [11,12]. Also, being a housewife or being widowed and divorced were most strickened [13].

In our study, the first approximation comprises the investigation the association between insomnia and selfesteem and the second approximation the association between insomnia and marital status in nursing staff in Primary Health Care, in Emergency Departments (ED) and in Intensive Care Units (ICU) of five major hospitals and 11 Health Centers in the island of Crete, Greece. In addition, no study has examined the role of insomnia symptoms compared with self - esteem and marital status in nursing personnel worldwide.

\section{General OBJECTIVE}

The general objective of the study was to investigate the insomnia in nursing staff in Primary Health Care, in Emergency Departments (ED) and in Intensive Care Units (ICU) of five major hospitals and 11 Health Centers in the prefecture of Crete.

\section{SPECIFIC ОBJECTIVES}

- To study the association between insomnia and self-esteem in nursing staff.

○ To study the association between insomnia and family status in nursing staff.

\section{SigNifiCANCE OF THE STUDY}

The present study is expected to be used by Human Resource Management of the Hospitals and Health Care Centers to design appropriate policies that can curb insomnia in nursing staff.

\section{METHODOLOGY}

The survey involved 213 nurses from five (5) hospitals and eleven (11) Health Centers in the island Crete. Of these, $24.9 \%$ were working at the Emergency Departments, while the other $45.5 \%$ were working in Intensive Care Units and 29.6\% in Primary Health Care. The present study was carried out from August 2017 to January 2018 and included the voluntary and anonymous participation of nursing staff. The psychometric tools included in the study are presented below: 
Association between Insomnia, Demographic Characteristics and Self - Esteem in Nursing Personnel in Primary Care and in Emergency - Intensive Care Units on the Island of Crete

\section{INSTRUMENTS}

For the purpose of the present research, the Hamilton Anxiety Scale (HAM-A) and the Culture-free Self-esteem Inventories were applied.

The Hamilton Anxiety Scale (HAM-A) was one of the first rating scales developed to measure the severity of anxiety symptoms, and is still widely used in both research and clinical settings. The scale consists of 14 statements, each defined by a series of symptoms, and measures psychic anxiety (mental agitation and psychological distress) and somatic anxiety (physical complaints related to anxiety). Scoring: Each item is scored on a scale of 0 (not present) to 4 (severe), with a total score range of $0-56$, where $<17$ indicates mild severity, 18-24 mild to moderate severity and 25-30 moderate to severe [14].

The Culture-free Self-esteem Inventories is a self-referencing questionnaire, which includes (without the lie scale) 32 items. These statements seek to measure the general, personal and social perception of themselves (self-perception) and are divided into two groups: those that are high and those showing low self-esteem. Each question answers either with "yes' or with "no". Three self-assessment sub-scales are included: general (16 statements), social (8 statements) and personal (8 statements). There is also a lie scale o (8 statements) [15].

Permissions were obtained from the developers of the Hamilton Anxiety Scale (HAM-A) and Culture-free Selfesteem Inventories. The time needed to fill out the questionnaire was 8-10 minutes.

\section{STUdy Population}

The present research conducted among nurses working in primary health-care setting with the participation of 14 Health-care Centers and 5 Hospitals in the island of Crete. Our sample consisted of 213 nurses take part in the study. The study was performed in the following departments: 14 Health-care Centers, 4 Emergency Departments (ED) and 11 Intensive Care Units (ICU). The study was performed during August 2017 to January 2018.

\section{INCLUSION CRITERIA}

- Selected participants had to work to Hospitals and Health Centers that had been included in the National Health System and had the same system of shift to ensure the homogeneity of the sample.

- Selected participants have to be nursing personnel with any educational level with each working relationship

\section{EXCLUSION CRITERIA}

- Participants had not to be nursing student

\section{Statistical ANalysis}

With respect to the statistical analysis, the quantitative variables are reported based on the mean \pm standard deviation (mean $\pm \mathrm{sd}$ ) as well as the median and the interquartile range (IQR), while for the qualitative variables we have the corresponding frequencies and percentages. Depending on the appropriate statistical and / or graphic controls, it is recommended that median and the interquartile range (median, IQR) are used as representative descriptive measures.

The appropriate parametric and non-parametric statistical checks were also performed to investigate any differences between the three structures (ICU, Primary Health Care and ED) and the scales under study, defining the materiality level at 0.05 . Where necessary, exact tests and / or Monte Carlo simulation (10000 samples) were used. 
Association between Insomnia, Demographic Characteristics and Self - Esteem in Nursing Personnel in Primary Care and in Emergency - Intensive Care Units on the Island of Crete

Finally, reliability and internal consistency was assessed by internal consistency using Cronbach 's alpha test. The statistical analysis was performed using statistical software IBM SPSS statistics (version 21.0). A p value $<0.05$ was considered statistically significant.

\section{ETHICS APPROVAL}

The necessary written permissions were issued from the Research and Ethics Committees of the 7th Health District of Crete, the University General Hospital of Heraklion, General Hospital of Heraklion "Venizelio Pananio", General Hospital of Chania, General Hospital of Rethymno and General Hospital of Agios Nikolaos. The results of the current study were announced in the Administration of the 7th Health District of Crete. Written consent was given by all participants to take part in the study.

\section{RESULTS}

\section{Characteristics of the Study Sample}

In the present study, the participants were nursing personnel $(n=213)$ and the majority of them $89.2 \%$ of the total sample were women and Intensive Care Units (ICU) nurses represented $45.5 \%$ of the study population. The mean age for the nursing staff of the total sample was 41.73 years. The $75.1 \%(n=160)$ of the sample were married. The majority of the total sample of the study $64.8 \%(n=138)$ was graduates of Technological Educational Institute and the $8.9 \%(n=18)$ had a master's degree. Demographic characteristics of the study population are shown in Table 1. The mean length of employment was 15.78 ( $\mathrm{SD}=8.49$ years). In regard of the average of work in the current department was 8.00 years ( $\mathrm{SD}=10.50$ years) Table 2.

Table1. Characteristics of the study population $(N=213)$.

\begin{tabular}{|c|c|c|}
\hline \multicolumn{2}{|l|}{ Characteristics } & n (\%) \\
\hline Departments & Intensive Care Unit & $97(45.5)$ \\
\hline & Primary Care & $63(29.6)$ \\
\hline Gender & Emergency Department & $53(24.9)$ \\
\hline & & $23(10.8)$ \\
\hline & Man & $190(89.2)$ \\
\hline Marital Status & Women & $160(75.1)$ \\
\hline & Married & $49(23.0)$ \\
\hline & Unmarried & $4(1.9)$ \\
\hline & Widowed/ Divorced & \\
\hline & & $138(64.8)$ \\
\hline & Secondary School & $13(6.1)$ \\
\hline & Technological Educational Institute & $18(8.4)$ \\
\hline & University & $1(0.5)$ \\
\hline
\end{tabular}


Association between Insomnia, Demographic Characteristics and Self - Esteem in Nursing Personnel in Primary Care and in Emergency - Intensive Care Units on the Island of Crete

Table2. Descriptive characteristics among the study population $(N=213)$.

\begin{tabular}{|c|c|}
\hline Characteristics & \\
\hline Age (years) & $41.73 \pm 7.33^{*}$ \\
\hline Years of work & $15.78 \pm 8.49^{*}$ \\
\hline Years of work in the current work position & $8.00(10.50)^{* *}$ \\
\hline
\end{tabular}

$*$ mean \pm sd

**median (IQR)

\section{Reliability of Culture-Free Self-Esteem Inventories (James Battle)}

Cronbach's $\alpha$ coefficient was 0.763 suggesting high internal consistency (General self-esteem: alpha $=0.737$, Social self-esteem: alpha $=0.442$, Personal self-esteem: alpha $=0.724$, General self-esteem: alpha $=0.763$ ).

\section{Valuation of Self-Esteem}

From the results of the Culture-free Self-esteem Inventories, it was found that $51.6 \%$ of the total sample who participated in the research had a middle self-esteem, while the lowest percentage $8.0 \%$ had very high selfesteem and the $8.0 \%$ had very low self-esteem. Also, it was found that $17.4 \%$ had low self-esteem and $15 \%$ had high self-esteem. Also, the results showed that the mean General self-esteem was 12.27, the mean Social selfesteem 6.35, the mean Personal self-esteem was 3.86 and the scale of lie was 4.77 as shown in Table 3. There are no statistically significant differences between the three departments Intensive Care Unit (ICU), Emergency Departments (ED) and Primary Care. Below are analyzed the level of self-esteem for the three departments (ICU, ED, Primary Care): ICU: very low self-esteem 8.2\%, low self-esteem $17.5 \%$, middle self-esteem 50.5\%, high self-esteem $13.4 \%$, very high self-esteem $10.3 \%$, ED: very low self-esteem $11.3 \%$, low self-esteem $26.4 \%$, middle self-esteem $37.7 \%$, high self-esteem $20.8 \%$, very high self-esteem $3.8 \%$, Primary Care: very low selfesteem: $4.8 \%$, low self-esteem $9.5 \%$, middle self-esteem $65.1 \%$, high self-esteem $12.7 \%$, very high self-esteem 7.9\% as shown in Table 4.

Table3. Descriptive characteristics of the scales among the study population $(N=213)$.

\begin{tabular}{|r|c|c|c|c|c|c|}
\hline Scales & $\mathbf{n}(\mathbf{6 )}$ & Mean & SD & Median & IQR & Range \\
\hline Culture-free Self-esteem Inventories (James Battle), & & & & & & \\
\hline General self-esteem * & & 12.27 & 2.89 & 13.00 & 3.50 & $2-16$ \\
\hline Social self-esteem * & & 6.35 & 1.38 & 7.00 & 1.00 & $1-8$ \\
\hline Personal self-esteem * & & 3.86 & 2.16 & 4.00 & 3.50 & $0-8$ \\
\hline Scale of lie & & 4.77 & 1.56 & 5.00 & 2.00 & $0-8$ \\
\hline Total self-esteem & & 22.47 & 5.28 & 23.00 & 7.00 & $4-32$ \\
\hline Too low & $17(8.0)$ & & & & & \\
\hline Low & $37(17.4)$ & & & & & \\
\hline Middle & $110(51.6)$ & & & & & \\
\hline Hugh & $32(15.0)$ & & & & & \\
\hline Very Hugh & $17(8.0)$ & & & & & \\
\hline
\end{tabular}

*(IQRs) as representative descriptive measures for these scales 
Association between Insomnia, Demographic Characteristics and Self - Esteem in Nursing Personnel in Primary Care and in Emergency - Intensive Care Units on the Island of Crete

Table4. Level of self-esteem among the study population $(N=213)$.

\begin{tabular}{|c|c|c|c|c|c|c|c|c|}
\hline & \multicolumn{5}{|c|}{ Level of self-esteem } & \multirow{2}{*}{ Total } \\
\hline & & & Very Low & Low & Middle & High & Very high & \\
\hline \multirow{6}{*}{ Department } & \multirow{2}{*}{ ICU } & $\mathrm{N}$ & 8 & 17 & 49 & 13 & 10 & 97 \\
\hline & & $\%$ & $8.2 \%$ & $17.5 \%$ & $50.5 \%$ & $13.4 \%$ & $10.3 \%$ & $100.0 \%$ \\
\hline & \multirow{2}{*}{$\begin{array}{c}\text { Primary } \\
\text { Care }\end{array}$} & $\mathrm{N}$ & 3 & 6 & 41 & 8 & 5 & 63 \\
\hline & & $\%$ & $4.8 \%$ & $9.5 \%$ & $65.1 \%$ & $12.7 \%$ & $7.9 \%$ & $100.0 \%$ \\
\hline & \multirow{2}{*}{ ED } & $\mathrm{N}$ & 6 & 14 & 20 & 11 & 2 & 53 \\
\hline & & $\%$ & $11.3 \%$ & $26.4 \%$ & $37.7 \%$ & $20.8 \%$ & $3.8 \%$ & $100.0 \%$ \\
\hline \multirow{2}{*}{\multicolumn{2}{|c|}{ Total }} & $\mathrm{N}$ & 17 & 37 & 110 & 32 & 17 & 213 \\
\hline & & $\%$ & $8.0 \%$ & $17.4 \%$ & $51.6 \%$ & $15.0 \%$ & $8.0 \%$ & $100.0 \%$ \\
\hline
\end{tabular}

$\chi^{2}(8)=13.898, p=0.080$

\section{Reliability of Hamilton Anxiety Scale (HAM-A)}

The reliability Of Hamilton Anxiety Scale (HAM-A) expressed by Cronbach $\alpha$ was 0.905 .

\section{Valuation of Intensity of the Symptoms Anxiety and Insomnia}

The intensity of the symptoms of anxiety had an average 11.41 based on their Hamilton Anxiety Estimation Scale. The most common mild intensity symptoms according Hamilton Anxiety Scale were, " anxious mood " (33.8\%), "tension" (32.4\%), "intellectual" (26.8\%), " somatic (muscular system)” (26.3\%), "insomnia" (23.9\%), "somatic (physical symptoms)" (23.5\%), "cardiovascular symptoms" (23.0\%), "depressed mood" (21.6\%), "gastrointestinal symptoms" $22.5 \%$, "fears" 20.7\%, "automatic symptoms" $20.2 \%$, "genitourinary symptoms " $17.8 \%$ and "respiratory symptoms" $16.9 \%$ ) as shown in Table 5.

The most common moderate intensity symptoms according Hamilton Anxiety Scale were, "anxious mood " (27.7\%), "tension" (17.8\%), "intellectual" (19.2.8\%), "somatic (muscular system)" (26.3\%), "insomnia" (23.9\%), "somatic (physical symptoms)" (23.5\%)," cardiovascular symptoms" (23.0\%), "depressed mood" (11.7\%), "gastrointestinal symptoms" $14.1 \%$, "fears" (7.5 \%), "automatic symptoms" (8.9\%), "genitourinary symptoms " (6.1\%) and "respiratory symptoms" (7.5\%) as shown in Table 5.

The most common severe intensity symptoms according Hamilton Anxiety Scale were, " anxious mood " (11.3\%), "tension" (12.2\%), "intellectual" (5.6\%), " somatic (muscular system)” (26.3\%), "insomnia” (23.9\%), "somatic (physical symptoms)" (9.9\%), "cardiovascular symptoms" (3.8\%), "depressed mood" (7.0\%), "gastrointestinal symptoms" (4.2\%), "fears" (4.2\%), "automatic symptoms" (4.2\%), "genitourinary symptoms "(3.8\%) and "respiratory symptoms" (4.2\%) as shown in Table 5.

The most common very severe intensity symptoms according Hamilton Anxiety Scale were, "anxious mood “ (3.3\%), "tension" (3.3\%), "intellectual" (3.3\%), " somatic (muscular system)" (6.1\%), "insomnia" (23.9\%), "somatic (physical symptoms)" (23.5\%), " cardiovascular symptoms " (2.3\%), "depressed mood" (7.0\%), "gastrointestinal symptoms" $3.3 \%$, "fears" (1.4\%), "automatic symptoms" (1.9\%), "genitourinary symptoms" $(1.4 \%)$ and "respiratory symptoms" (1.9\%) as shown in Table 5.

In our sample, $49.7 \%$ of the nursing personnel had possible insomnia based on their Hamilton Anxiety Estimation Scale of the overall study sample. The $23.9 \%$ of the total sample had mild insomnia, the $15.5 \%$ had moderate insomnia, the $6.6 \%$ had severe insomnia and the $3.3 \%$ had very severe insomnia as shown in Table 5. 
Association between Insomnia, Demographic Characteristics and Self - Esteem in Nursing Personnel in Primary Care and in Emergency - Intensive Care Units on the Island of Crete

Table5. Hamilton Anxiety Estimation Scale of the overall study sample $(N=213)$.

\begin{tabular}{|c|l|c|c|c|c|c|}
\hline & & None & Mild & Moderate & Severe & Very severe \\
\hline & \multicolumn{5}{|c|}{ \% } \\
\hline 1. & Anxious mood & 23.9 & 33.8 & 27.7 & 11.3 & 3.3 \\
\hline 2. & Tension & 34.7 & 32.4 & 17.8 & 12.2 & 2.8 \\
\hline 3. & Fears & 66.2 & 20.7 & 7.5 & 4.2 & 1.4 \\
\hline $\mathbf{4}$ & Insomnia & $\mathbf{5 0 . 7}$ & $\mathbf{2 3 . 9}$ & $\mathbf{1 5 . 5}$ & $\mathbf{6 . 6}$ & $\mathbf{3 . 3}$ \\
\hline $\mathbf{5 .}$ & Intellectual & 45.1 & 26.8 & 19.2 & 5.6 & 3.3 \\
\hline $\mathbf{6 .}$ & Depressed mood & 55.9 & 21.6 & 11.7 & 7.0 & 3.8 \\
\hline 7. & Somatic (muscular) & 41.3 & 26.3 & 16.4 & 9.9 & 6.1 \\
\hline 8. & Somatic (sensory) & 59.2 & 23.5 & 9.9 & 4.7 & 2.8 \\
\hline 9. & Cardiovascular symptoms & 60.6 & 23.0 & 10.3 & 3.8 & 2.3 \\
\hline $\mathbf{1 0 .}$ & Respiratory symptoms & 69.5 & 16.9 & 7.5 & 4.2 & 1.9 \\
\hline $\mathbf{1 1 .}$ & Gastrointestinal symptoms & 54.5 & 22.5 & 14.1 & 5.6 & 3.3 \\
\hline $\mathbf{1 2 .}$ & Genitourinary symptoms & 70.9 & 17.8 & 6.1 & 3.8 & 1.4 \\
\hline $\mathbf{1 3 .}$ & Autonomic symptoms & 64.8 & 20.2 & 8.9 & 4.2 & 1.9 \\
\hline
\end{tabular}

Association of Insomnia with Self-Esteem and Demographic Characteristics

We calculate the observed p-value with the Monte Carlo (2-sided) simulation method. Multiple regression analyses were performed to examine association between insomnia and marital status Table 6. Moreover, analyses were performed to examine association between insomnia and self-esteem as shown in Table 7. As shown in Table 6, Table 7 marital status and self-esteem were significant predictors of insomnia. Results verified the significant positive association between insomnia and self - esteem. Also, results verified the significant positive association between insomnia and marital status.

Results verified the significant negative association between insomnia and education level ( $\mathrm{p}=0.973)$, age $(\mathrm{p}=0.751)$, gender $(\mathrm{p}=0.162)$, work experience $(\mathrm{p}=0.460)$ and work experience years in the current department/ workplace $(\mathrm{p}=0.834)$.

Table6. Association between insomnia and family status in the nursing staff of the overall study sample $(N=213)$.

\begin{tabular}{|c|c|c|c|c|c|c|}
\hline \multicolumn{7}{|c|}{ Crosstab } \\
\hline & & & \multicolumn{3}{|c|}{ Family status } & \multirow{2}{*}{ Total } \\
\hline & & & Married & Unmarried & Widowed/Divorced & \\
\hline \multirow{15}{*}{ Insomnia } & \multirow{3}{*}{ None } & Count & 87 & 18 & 3 & 108 \\
\hline & & $\%$ within insomnia & $80.6 \%$ & $16.7 \%$ & $2.8 \%$ & $100.0 \%$ \\
\hline & & \% within Family status & $54.4 \%$ & $36.7 \%$ & $75.0 \%$ & $50.7 \%$ \\
\hline & \multirow{3}{*}{ Mild } & Count & 42 & 8 & 1 & 51 \\
\hline & & \% within insomnia & $82.4 \%$ & $15.7 \%$ & $2.0 \%$ & $100.0 \%$ \\
\hline & & $\%$ within Family status & $26.3 \%$ & $16.3 \%$ & $25.0 \%$ & $23.9 \%$ \\
\hline & \multirow{3}{*}{ Moderate } & Count & 17 & 16 & 0 & 33 \\
\hline & & $\%$ within insomnia & $51.5 \%$ & $48.5 \%$ & $0.0 \%$ & $100.0 \%$ \\
\hline & & $\%$ within Family status & $10.6 \%$ & $32.7 \%$ & $0.0 \%$ & $15.5 \%$ \\
\hline & \multirow{3}{*}{ Severe } & Count & 9 & 5 & 0 & 14 \\
\hline & & $\%$ within insomnia & $64.3 \%$ & $35.7 \%$ & $0.0 \%$ & $100.0 \%$ \\
\hline & & \% within Family status & $5.6 \%$ & $10.2 \%$ & $0.0 \%$ & $6.6 \%$ \\
\hline & \multirow{3}{*}{ Very Severe } & Count & 5 & 2 & 0 & 7 \\
\hline & & \% within insomnia & $71.4 \%$ & $28.6 \%$ & $0.0 \%$ & $100.0 \%$ \\
\hline & & \% within Family status & $3.1 \%$ & $4.1 \%$ & $0.0 \%$ & $3.3 \%$ \\
\hline
\end{tabular}

American Research Journal of Nursing

Page 7 
Association between Insomnia, Demographic Characteristics and Self - Esteem in Nursing Personnel in Primary Care and in Emergency - Intensive Care Units on the Island of Crete

\begin{tabular}{|c|c|c|c|c|c|c|c|c|c|c|}
\hline \multirow{3}{*}{ Total } & \multicolumn{4}{|c|}{ Count } & 160 & \multicolumn{2}{|c|}{49} & \multicolumn{2}{|c|}{4} & 213 \\
\hline & \multicolumn{4}{|c|}{$\%$ within insomnia } & $75.1 \%$ & \multicolumn{2}{|c|}{$23.0 \%$} & \multicolumn{2}{|c|}{$1.9 \%$} & $100.0 \%$ \\
\hline & \multicolumn{4}{|c|}{$\%$ within Family status } & $100.0 \%$ & \multicolumn{2}{|c|}{$100.0 \%$} & \multicolumn{2}{|c|}{$100.0 \%$} & $100.0 \%$ \\
\hline \multicolumn{11}{|c|}{ Chi-Square Tests } \\
\hline & \multirow{3}{*}{\multicolumn{2}{|c|}{ Value }} & \multirow{3}{*}{ dff } & \multirow{3}{*}{$\begin{array}{l}\text { Asymp. Sig. } \\
\text { (2-sided) }\end{array}$} & \multicolumn{3}{|c|}{ Monte Carlo Sig. (2-sided) } & \multicolumn{3}{|c|}{ Monte Carlo Sig. (1-sided) } \\
\hline & & & & & \multirow{2}{*}{ Sig. } & \multicolumn{2}{|c|}{$\begin{array}{c}\text { 99\% Confidence } \\
\text { Interval }\end{array}$} & \multirow{2}{*}{ Sig. } & \multicolumn{2}{|c|}{$\begin{array}{c}\text { 99\% Confidence } \\
\text { Interval } \\
\end{array}$} \\
\hline & & & & & & $\begin{array}{l}\text { Lower } \\
\text { Bound }\end{array}$ & $\begin{array}{l}\text { Upper } \\
\text { Bound }\end{array}$ & & $\begin{array}{l}\text { Lower } \\
\text { Bound }\end{array}$ & $\begin{array}{l}\text { Upper } \\
\text { Bound }\end{array}$ \\
\hline Pearson Chi-Square & & $18.405^{\mathrm{a}}$ & 8 & .018 & $.026^{\mathrm{b}}$ & .022 & .030 & & & \\
\hline Likelihood Ratio & & 17.586 & 8 & .025 & $.020^{\mathrm{b}}$ & .016 & .024 & & & \\
\hline Fisher's Exact Test & & 16.976 & & & $.014^{\mathrm{b}}$ & .011 & .017 & & & \\
\hline \multicolumn{2}{|l|}{ Linear-by-Linear Association } & $3.712^{\mathrm{c}}$ & 1 & .054 & $.058^{\mathrm{b}}$ & .052 & .064 & $.033^{\mathrm{b}}$ & .028 & .038 \\
\hline $\mathrm{N}$ of Valid Cases & & 213 & & & & & & & & \\
\hline
\end{tabular}

a. 7 cells (46.7\%) have expected count less than 5 . The minimum expected count is .13.

b. Based on 10000 sampled tables with starting seed 423042446.

c. The standardized statistic is 1.927 .

Table7. Association between insomnia and total self-esteem in the nursing staff of the overall study sample ( $N=213)$.

\begin{tabular}{|c|c|c|c|c|c|c|c|c|}
\hline \multicolumn{9}{|c|}{ Crosstab } \\
\hline & & & \multicolumn{5}{|c|}{ Self - esteem } & \multirow{2}{*}{ Total } \\
\hline & & & Very Low & Low & Middle & High & Very high & \\
\hline \multirow{15}{*}{ Insomnia } & \multirow{3}{*}{ None } & Count & 3 & 13 & 58 & 20 & 14 & 108 \\
\hline & & $\%$ within insomnia & $2.8 \%$ & $12.0 \%$ & $53.7 \%$ & $18.5 \%$ & $13.0 \%$ & $100.0 \%$ \\
\hline & & $\%$ within Self-esteem & $17.6 \%$ & $35.1 \%$ & $52.7 \%$ & $62.5 \%$ & $82.4 \%$ & $50.7 \%$ \\
\hline & \multirow{3}{*}{ Mild } & Count & 3 & 11 & 26 & 9 & 2 & 51 \\
\hline & & $\%$ within insomnia & $5.9 \%$ & $21.6 \%$ & $51.0 \%$ & $17.6 \%$ & $3.9 \%$ & $100.0 \%$ \\
\hline & & $\%$ within Self-esteem & $17.6 \%$ & $29.7 \%$ & $23.6 \%$ & $28.1 \%$ & $11.8 \%$ & $23.9 \%$ \\
\hline & \multirow{3}{*}{ Moderate } & Count & 6 & 6 & 18 & 2 & 1 & 33 \\
\hline & & \% within insomnia & $18.2 \%$ & $18.2 \%$ & $54.5 \%$ & $6.1 \%$ & $3.0 \%$ & $100.0 \%$ \\
\hline & & $\%$ within Self-esteem & $35.3 \%$ & $16.2 \%$ & $16.4 \%$ & $6.3 \%$ & $5.9 \%$ & $15.5 \%$ \\
\hline & \multirow{3}{*}{ Severe } & Count & 2 & 5 & 6 & 1 & 0 & 14 \\
\hline & & $\%$ within insomnia & $14.3 \%$ & $35.7 \%$ & $42.9 \%$ & $7.1 \%$ & $0.0 \%$ & $100.0 \%$ \\
\hline & & $\%$ within Self-esteem & $11.8 \%$ & $13.5 \%$ & $5.5 \%$ & $3.1 \%$ & $0.0 \%$ & $6.6 \%$ \\
\hline & \multirow{3}{*}{ Very Severe } & Count & 3 & 2 & 2 & 0 & 0 & 7 \\
\hline & & $\%$ within insomnia & $42.9 \%$ & $28.6 \%$ & $28.6 \%$ & $0.0 \%$ & $0.0 \%$ & $100.0 \%$ \\
\hline & & $\%$ within Self-esteem & $17.6 \%$ & $5.4 \%$ & $1.8 \%$ & $0.0 \%$ & $0.0 \%$ & $3.3 \%$ \\
\hline \multirow{3}{*}{\multicolumn{2}{|c|}{ Total }} & Count & 17 & 37 & 110 & 32 & 17 & 213 \\
\hline & & $\%$ within insomnia & $8.0 \%$ & $17.4 \%$ & $51.6 \%$ & $15.0 \%$ & $8.0 \%$ & $100.0 \%$ \\
\hline & & \% within Self-esteem & $100.0 \%$ & $100.0 \%$ & $100.0 \%$ & $100.0 \%$ & $100.0 \%$ & $100.0 \%$ \\
\hline
\end{tabular}

American Research Journal of Nursing 
Association between Insomnia, Demographic Characteristics and Self - Esteem in Nursing Personnel in Primary Care and in Emergency - Intensive Care Units on the Island of Crete

\begin{tabular}{|c|c|c|c|c|c|c|c|c|c|}
\hline \multicolumn{10}{|c|}{ Chi-Square Tests } \\
\hline & \multirow{3}{*}{ Value } & \multirow{3}{*}{ df } & \multirow{3}{*}{$\begin{array}{c}\text { Asymp. Sig } \\
\text { (2-sided) }\end{array}$} & \multicolumn{3}{|c|}{ Monte Carlo Sig. (2-sided) } & \multicolumn{3}{|c|}{ Monte Carlo Sig. (1-sided) } \\
\hline & & & & \multirow{2}{*}{ Sig. } & \multicolumn{2}{|c|}{$\begin{array}{l}\text { 99\% Confidence } \\
\text { Interval }\end{array}$} & \multirow{2}{*}{ Sig. } & \multicolumn{2}{|c|}{$\begin{array}{l}\text { 99\% Confidence } \\
\text { Interval }\end{array}$} \\
\hline & & & & & $\begin{array}{l}\text { Lower } \\
\text { Bound }\end{array}$ & $\begin{array}{l}\text { Upper } \\
\text { Bound }\end{array}$ & & $\begin{array}{l}\text { Lower } \\
\text { Bound }\end{array}$ & $\begin{array}{l}\text { Upper } \\
\text { Bound }\end{array}$ \\
\hline Pearson Chi-Square & $37.814^{\mathrm{a}}$ & 16 & .002 & $.002^{b}$ & .001 & .004 & & & \\
\hline Likelihood Ratio & 35.276 & 16 & .004 & $.006^{\mathrm{b}}$ & .004 & .008 & & & \\
\hline Fisher's Exact Test & 30.879 & & & $.004^{\mathrm{b}}$ & .002 & .006 & & & \\
\hline Linear-by-Linear Association & $28.165^{c}$ & 1 & .000 & $.000^{\mathrm{b}}$ & .000 & .000 & $.000^{\mathrm{b}}$ & .000 & .000 \\
\hline $\mathrm{N}$ of Valid Cases & 213 & & & & & & & & \\
\hline
\end{tabular}

Table8. Association between insomnia and education level in the nursing staff of the overall study sample ( $N=213$ )

\begin{tabular}{|c|c|c|c|c|c|c|c|}
\hline \multicolumn{8}{|c|}{ Crosstab } \\
\hline & & & \multicolumn{4}{|c|}{ Educational level } & \multirow[b]{2}{*}{ Total } \\
\hline & & & $\begin{array}{c}\text { Secondary } \\
\text { School }\end{array}$ & $\begin{array}{c}\text { Higher } \\
\text { technological } \\
\text { education }\end{array}$ & University & $\begin{array}{l}\text { Master } \\
\text { degree \& } \\
\text { PhD }\end{array}$ & \\
\hline \multirow{15}{*}{ Insomnia } & \multirow{3}{*}{ None } & Count & 22 & 70 & 5 & 11 & 108 \\
\hline & & $\%$ within insomnia & $20.4 \%$ & $64.8 \%$ & $4.6 \%$ & $10.2 \%$ & $100.0 \%$ \\
\hline & & $\%$ within educational level & $51.2 \%$ & $50.7 \%$ & $38.5 \%$ & $57.9 \%$ & $50.7 \%$ \\
\hline & \multirow{3}{*}{ Mild } & Count & 11 & 31 & 4 & 5 & 51 \\
\hline & & $\%$ within insomnia & $21.6 \%$ & $60.8 \%$ & $7.8 \%$ & $9.8 \%$ & $100.0 \%$ \\
\hline & & $\%$ within education level & $25.6 \%$ & $22.5 \%$ & $30.8 \%$ & $26.3 \%$ & $23.9 \%$ \\
\hline & \multirow{3}{*}{ Moderate } & Count & 6 & 22 & 3 & 2 & 33 \\
\hline & & $\%$ within insomnia & $18.2 \%$ & $66.7 \%$ & $9.1 \%$ & $6.1 \%$ & $100.0 \%$ \\
\hline & & $\%$ within education level & $14.0 \%$ & $15.9 \%$ & $23.1 \%$ & $10.5 \%$ & $15.5 \%$ \\
\hline & \multirow{3}{*}{ Severe } & Count & 2 & 11 & 1 & 0 & 14 \\
\hline & & $\%$ within insomnia & $14.3 \%$ & $78.6 \%$ & $7.1 \%$ & $0.0 \%$ & $100.0 \%$ \\
\hline & & $\%$ within educational level & $4.7 \%$ & $8.0 \%$ & $7.7 \%$ & $0.0 \%$ & $6.6 \%$ \\
\hline & \multirow{3}{*}{ Very severe } & Count & 2 & 4 & 0 & 1 & 7 \\
\hline & & $\%$ within insomnia & $28.6 \%$ & $57.1 \%$ & $0.0 \%$ & $14.3 \%$ & $100.0 \%$ \\
\hline & & $\%$ within education level & $4.7 \%$ & $2.9 \%$ & $0.0 \%$ & $5.3 \%$ & $3.3 \%$ \\
\hline \multirow{3}{*}{\multicolumn{2}{|c|}{ Total }} & Count & 43 & 138 & 13 & 19 & 213 \\
\hline & & $\%$ within insomnia & $20.2 \%$ & $64.8 \%$ & $6.1 \%$ & $8.9 \%$ & $100.0 \%$ \\
\hline & & $\%$ within educational level & $100.0 \%$ & $100.0 \%$ & $100.0 \%$ & $100.0 \%$ & $100.0 \%$ \\
\hline
\end{tabular}


Association between Insomnia, Demographic Characteristics and Self - Esteem in Nursing Personnel in Primary Care and in Emergency - Intensive Care Units on the Island of Crete

\begin{tabular}{|c|c|c|c|c|c|c|c|c|c|}
\hline \multicolumn{10}{|c|}{ Chi-Square Tests } \\
\hline & \multirow{3}{*}{ Value } & \multirow{3}{*}{ df } & \multirow{3}{*}{$\begin{array}{c}\text { Asymp. } \\
\text { Sig. } \\
\text { (2-sided) }\end{array}$} & \multicolumn{3}{|c|}{ Monte Carlo Sig. (2-sided) } & \multicolumn{3}{|c|}{ Monte Carlo Sig. (1-sided) } \\
\hline & & & & \multirow{2}{*}{ Sig. } & \multicolumn{2}{|c|}{$\begin{array}{c}\text { 99\% Confidence } \\
\text { Interval }\end{array}$} & \multirow{2}{*}{ Sig. } & \multicolumn{2}{|c|}{$\begin{array}{c}\text { 99\% Confidence } \\
\text { Interval }\end{array}$} \\
\hline & & & & & $\begin{array}{l}\text { Lower } \\
\text { Bound }\end{array}$ & $\begin{array}{l}\text { Upper } \\
\text { Bound }\end{array}$ & & $\begin{array}{l}\text { Lower } \\
\text { Bound }\end{array}$ & $\begin{array}{l}\text { Upper } \\
\text { Bound }\end{array}$ \\
\hline Pearson Chi-Square & $4.806^{\mathrm{a}}$ & 12 & .964 & $.973^{\mathrm{b}}$ & .969 & .978 & & & \\
\hline Likelihood Ratio & 6.405 & 12 & .894 & $.949^{\mathrm{b}}$ & .943 & .955 & & & \\
\hline Fisher's Exact Test & 5.006 & & & $.960^{\mathrm{b}}$ & .955 & .965 & & & \\
\hline Linear-by-Linear Association & $.158^{c}$ & 1 & .691 & $.692^{\mathrm{b}}$ & .681 & .704 & $.380^{\mathrm{b}}$ & .367 & .392 \\
\hline $\mathrm{N}$ of Valid Cases & 213 & & & & & & & & \\
\hline
\end{tabular}

a. 11 cells (55.0\%) have expected count less than 5 . The minimum expected count is .43 .

b. Based on 10000 sampled tables with starting seed 423042446.

c. The standardized statistic is -.398 .

Table9. Association between insomnia and age in the nursing staff of the overall study sample $(N=213)$.

\begin{tabular}{|c|c|c|c|c|c|c|}
\hline \multicolumn{7}{|c|}{ Crosstab } \\
\hline & & & \multicolumn{3}{|c|}{ age } & \multirow{2}{*}{ Total } \\
\hline & & & $<=30$ & $31-45$ & $46+$ & \\
\hline \multirow{15}{*}{ Insomnia } & \multirow{3}{*}{ None } & Count & 6 & 60 & 42 & 108 \\
\hline & & $\%$ within insomnia & $5.6 \%$ & $55.6 \%$ & $38.9 \%$ & $100.0 \%$ \\
\hline & & $\%$ within age & $50.0 \%$ & $47.2 \%$ & $56.8 \%$ & $50.7 \%$ \\
\hline & \multirow{3}{*}{ Mild } & Count & 3 & 33 & 15 & 51 \\
\hline & & $\%$ within insomnia & $5.9 \%$ & $64.7 \%$ & $29.4 \%$ & $100.0 \%$ \\
\hline & & $\%$ within age & $25.0 \%$ & $26.0 \%$ & $20.3 \%$ & $23.9 \%$ \\
\hline & \multirow{3}{*}{ Moderate } & Count & 3 & 19 & 11 & 33 \\
\hline & & $\%$ within insomnia & $9.1 \%$ & $57.6 \%$ & $33.3 \%$ & $100.0 \%$ \\
\hline & & $\%$ within age & $25.0 \%$ & $15.0 \%$ & $14.9 \%$ & $15.5 \%$ \\
\hline & \multirow{3}{*}{ Severe } & Count & 0 & 11 & 3 & 14 \\
\hline & & $\%$ within insomnia & $0.0 \%$ & $78.6 \%$ & $21.4 \%$ & $100.0 \%$ \\
\hline & & $\%$ within age & $0.0 \%$ & $8.7 \%$ & $4.1 \%$ & $6.6 \%$ \\
\hline & \multirow{3}{*}{ Very severe } & Count & 0 & 4 & 3 & 7 \\
\hline & & $\%$ within insomnia & $0.0 \%$ & $57.1 \%$ & $42.9 \%$ & $100.0 \%$ \\
\hline & & $\%$ within age & $0.0 \%$ & $3.1 \%$ & $4.1 \%$ & $3.3 \%$ \\
\hline \multirow{3}{*}{\multicolumn{2}{|c|}{ Total }} & Count & 12 & 127 & 74 & 213 \\
\hline & & $\%$ within insomnia & $5.6 \%$ & $59.6 \%$ & $34.7 \%$ & $100.0 \%$ \\
\hline & & $\%$ within age & $100.0 \%$ & $100.0 \%$ & $100.0 \%$ & $100.0 \%$ \\
\hline
\end{tabular}


Association between Insomnia, Demographic Characteristics and Self - Esteem in Nursing Personnel in Primary Care and in Emergency - Intensive Care Units on the Island of Crete

\begin{tabular}{|c|c|c|c|c|c|c|c|c|c|}
\hline \multicolumn{10}{|c|}{ Chi-Square Tests } \\
\hline & \multirow{3}{*}{ Value } & \multirow{3}{*}{ df } & \multirow{3}{*}{$\begin{array}{l}\text { Asymp. } \\
\text { Sig. } \\
\text { (2-sided) }\end{array}$} & \multicolumn{3}{|c|}{ Monte Carlo Sig. (2-sided) } & \multicolumn{3}{|c|}{ Monte Carlo Sig. (1-sided) } \\
\hline & & & & \multirow{2}{*}{ Sig. } & \multicolumn{2}{|c|}{$\begin{array}{c}\text { 99\% Confidence } \\
\text { Interval }\end{array}$} & \multirow{2}{*}{ Sig. } & \multicolumn{2}{|c|}{$\begin{array}{c}\text { 99\% Confidence } \\
\text { Interval }\end{array}$} \\
\hline & & & & & $\begin{array}{l}\text { Lower } \\
\text { Bound }\end{array}$ & $\begin{array}{l}\text { Upper } \\
\text { Bound }\end{array}$ & & $\begin{array}{l}\text { Lower } \\
\text { Bound }\end{array}$ & $\begin{array}{l}\text { Upper } \\
\text { Bound }\end{array}$ \\
\hline Pearson Chi-Square & $5.102^{2}$ & 8 & .747 & $.751^{\mathrm{b}}$ & .740 & .762 & & & \\
\hline Likelihood Ratio & 6.206 & 8 & .624 & $.696^{\mathrm{b}}$ & .684 & .708 & & & \\
\hline Fisher's Exact Test & 4.337 & & & $.809^{\mathrm{b}}$ & .798 & .819 & & & \\
\hline Linear-by-Linear Association & $.358^{\mathrm{c}}$ & 1 & .549 & $.583^{\mathrm{b}}$ & .570 & .596 & $.294^{\mathrm{b}}$ & .282 & .306 \\
\hline $\mathrm{N}$ of Valid Cases & 213 & & & & & & & & \\
\hline
\end{tabular}

a. 7 cells (46.7\%) have expected count less than 5 . The minimum expected count is .39 .

b. Based on 10000 sampled tables with starting seed 423042446.

c. The standardized statistic is -..599.

Table10. Association between insomnia and gender in the nursing staff of the overall study sample $(N=213)$

\begin{tabular}{|c|c|c|c|c|c|}
\hline \multicolumn{6}{|c|}{ Crosstab } \\
\hline & & & \multicolumn{2}{|c|}{ Gender } & \multirow{2}{*}{ Total } \\
\hline & & & Male & Female & \\
\hline \multirow{15}{*}{ Insomnia } & \multirow{3}{*}{ None } & Count & 14 & 94 & 108 \\
\hline & & $\%$ within insomnia & $13.0 \%$ & $87.0 \%$ & $100.0 \%$ \\
\hline & & $\%$ within gender & $60.9 \%$ & $49.5 \%$ & $50.7 \%$ \\
\hline & \multirow{3}{*}{ Mild } & Count & 8 & 43 & 51 \\
\hline & & $\%$ within insomnia & $15.7 \%$ & $84.3 \%$ & $100.0 \%$ \\
\hline & & $\%$ within gender & $34.8 \%$ & $22.6 \%$ & $23.9 \%$ \\
\hline & \multirow{3}{*}{ Moderate } & Count & 1 & 32 & 33 \\
\hline & & $\%$ within insomnia & $3.0 \%$ & $97.0 \%$ & $100.0 \%$ \\
\hline & & $\%$ within gender & $4.3 \%$ & $16.8 \%$ & $15.5 \%$ \\
\hline & \multirow{3}{*}{ Severe } & Count & 0 & 14 & 14 \\
\hline & & $\%$ within insomnia & $0.0 \%$ & $100.0 \%$ & $100.0 \%$ \\
\hline & & $\%$ within gender & $0.0 \%$ & $7.4 \%$ & $6.6 \%$ \\
\hline & \multirow{3}{*}{ Very severe } & Count & 0 & 7 & 7 \\
\hline & & $\%$ within insomnia & $0.0 \%$ & $100.0 \%$ & $100.0 \%$ \\
\hline & & $\%$ within gender & $0.0 \%$ & $3.7 \%$ & $3.3 \%$ \\
\hline \multirow{3}{*}{\multicolumn{2}{|c|}{ Total }} & Count & 23 & 190 & 213 \\
\hline & & $\%$ within insomnia & $10.8 \%$ & $89.2 \%$ & $100.0 \%$ \\
\hline & & $\%$ within gender & $100.0 \%$ & $100.0 \%$ & $100.0 \%$ \\
\hline
\end{tabular}


Association between Insomnia, Demographic Characteristics and Self - Esteem in Nursing Personnel in Primary Care and in Emergency - Intensive Care Units on the Island of Crete

\begin{tabular}{|c|c|c|c|c|c|c|c|c|c|}
\hline \multicolumn{10}{|c|}{ Chi-Square Tests } \\
\hline & \multirow{3}{*}{ Value } & \multirow{3}{*}{ df } & \multirow{3}{*}{$\begin{array}{l}\text { Asymp. } \\
\text { Sig. } \\
\text { (2-sided) }\end{array}$} & \multicolumn{3}{|c|}{ Monte Carlo Sig. (2-sided) } & \multicolumn{3}{|c|}{ Monte Carlo Sig. (1-sided) } \\
\hline & & & & \multirow{2}{*}{ Sig. } & \multicolumn{2}{|c|}{$\begin{array}{c}\text { 99\% Confidence } \\
\text { Interval }\end{array}$} & \multirow{2}{*}{ Sig. } & \multicolumn{2}{|c|}{$\begin{array}{c}\text { 99\% Confidence } \\
\text { Interval }\end{array}$} \\
\hline & & & & & $\begin{array}{l}\text { Lower } \\
\text { Bound }\end{array}$ & $\begin{array}{l}\text { Upper } \\
\text { Bound }\end{array}$ & & $\begin{array}{l}\text { Lower } \\
\text { Bound }\end{array}$ & $\begin{array}{l}\text { Upper } \\
\text { Bound }\end{array}$ \\
\hline Pearson Chi-Square & $6.400^{\mathrm{a}}$ & 4 & .171 & $.162^{\mathrm{b}}$ & .153 & .171 & & & \\
\hline Likelihood Ratio & 9.227 & 4 & .056 & $.064^{\mathrm{b}}$ & .058 & .070 & & & \\
\hline Fisher's Exact Test & 5.123 & & & $.227^{\mathrm{b}}$ & .216 & .238 & & & \\
\hline Linear-by-Linear Association & $4.180^{\circ}$ & 1 & .041 & $.044^{\mathrm{b}}$ & .039 & .049 & $.021^{\mathrm{b}}$ & .017 & .025 \\
\hline $\mathrm{N}$ of Valid Cases & 213 & & & & & & & & \\
\hline
\end{tabular}

a. 3 cells (30.0\%) have expected count less than 5. The minimum expected count is 76 .

b. Based on 10000 sampled tables with starting seed 423042446.

c. The standardized statistic is 2.044 .

Table11. Association between insomnia and years of work experience in the nursing staff of the overall study sample $(N=213)$.

\begin{tabular}{|c|c|c|c|c|c|c|}
\hline \multicolumn{7}{|c|}{ Crosstab } \\
\hline & & & \multicolumn{3}{|c|}{ Years of work experience } & \multirow{2}{*}{ Total } \\
\hline & & & $<=5$ & $6-10$ & $11+$ & \\
\hline \multirow{15}{*}{ Insomnia } & \multirow{3}{*}{ None } & Count & 8 & 25 & 75 & 108 \\
\hline & & $\%$ within insomnia & $7.4 \%$ & $23.1 \%$ & $69.4 \%$ & $100.0 \%$ \\
\hline & & $\%$ within years of work experience & $42.1 \%$ & $47.2 \%$ & $53.2 \%$ & $50.7 \%$ \\
\hline & \multirow{3}{*}{ Mild } & Count & 4 & 13 & 34 & 51 \\
\hline & & $\%$ within insomnia & $7.8 \%$ & $25.5 \%$ & $66.7 \%$ & $100.0 \%$ \\
\hline & & $\%$ years of work experience & $21.1 \%$ & $24.5 \%$ & $24.1 \%$ & $23.9 \%$ \\
\hline & \multirow{3}{*}{ Moderate } & Count & 5 & 6 & 22 & 33 \\
\hline & & $\%$ within insomnia & $15.2 \%$ & $18.2 \%$ & $66.7 \%$ & $100.0 \%$ \\
\hline & & $\%$ years of work experience & $26.3 \%$ & $11.3 \%$ & $15.6 \%$ & $15.5 \%$ \\
\hline & \multirow{3}{*}{ Severe } & Count & 2 & 6 & 6 & 14 \\
\hline & & $\%$ within insomnia & $14.3 \%$ & $42.9 \%$ & $42.9 \%$ & $100.0 \%$ \\
\hline & & $\%$ years of work experience & $10.5 \%$ & $11.3 \%$ & $4.3 \%$ & $6.6 \%$ \\
\hline & \multirow{3}{*}{ Very Severe } & Count & 0 & 3 & 4 & 7 \\
\hline & & $\%$ within insomnia & $0.0 \%$ & $42.9 \%$ & $57.1 \%$ & $100.0 \%$ \\
\hline & & $\%$ years of work experience & $0.0 \%$ & $5.7 \%$ & $2.8 \%$ & $3.3 \%$ \\
\hline \multirow{3}{*}{\multicolumn{2}{|c|}{ Total }} & Count & 19 & 53 & 141 & 213 \\
\hline & & $\%$ within insomnia & $8.9 \%$ & $24.9 \%$ & $66.2 \%$ & $100.0 \%$ \\
\hline & & $\%$ within years of work experience & $100.0 \%$ & $100.0 \%$ & $100.0 \%$ & $100.0 \%$ \\
\hline
\end{tabular}


Association between Insomnia, Demographic Characteristics and Self - Esteem in Nursing Personnel in Primary Care and in Emergency - Intensive Care Units on the Island of Crete

\begin{tabular}{|c|c|c|c|c|c|c|c|c|c|}
\hline \multicolumn{10}{|c|}{ Chi-Square Tests } \\
\hline & \multirow{3}{*}{ Value } & \multirow{3}{*}{$\mathbf{d f}$} & \multirow{3}{*}{$\begin{array}{l}\text { Asymp. Sig. } \\
\text { (2-sided) }\end{array}$} & \multicolumn{3}{|c|}{ Monte Carlo Sig. (2-sided) } & \multicolumn{3}{|c|}{ Monte Carlo Sig. (1-sided) } \\
\hline & & & & \multirow{2}{*}{ Sig. } & \multicolumn{2}{|c|}{$\begin{array}{c}\text { 99\% Confidence } \\
\text { Interval }\end{array}$} & \multirow{2}{*}{ Sig. } & \multicolumn{2}{|c|}{$\begin{array}{c}\text { 99\% Confidence } \\
\text { Interval }\end{array}$} \\
\hline & & & & & $\begin{array}{l}\text { Lower } \\
\text { Bound }\end{array}$ & $\begin{array}{l}\text { Upper } \\
\text { Bound }\end{array}$ & & $\begin{array}{l}\text { Lower } \\
\text { Bound }\end{array}$ & Upper Bound \\
\hline Pearson Chi-Square & $7.730^{\mathrm{a}}$ & 8 & .460 & $.460^{\mathrm{b}}$ & .447 & .473 & & & \\
\hline Likelihood Ratio & 7.796 & 8 & .454 & $.535^{\mathrm{b}}$ & .522 & .548 & & & \\
\hline Fisher's Exact Test & 7.656 & & & $.427^{\mathrm{b}}$ & .414 & .439 & & & \\
\hline Linear-by-Linear Association & $2.091^{\mathrm{c}}$ & 1 & .148 & $.157^{\mathrm{b}}$ & .147 & .166 & $.090^{\mathrm{b}}$ & .083 & .097 \\
\hline $\mathrm{N}$ of Valid Cases & 213 & & & & & & & & \\
\hline
\end{tabular}

a. 7 cells (46.7\%) have expected count less than 5. The minimum expected count is .62.

b. Based on 10000 sampled tables with starting seed 423042446.

c. The standardized statistic is - -437 .

Table12. Association between insomnia and years of work experience in the current work position in the nursing staff of the overall study sample $(N=213)$.

\begin{tabular}{|c|c|c|c|c|c|c|}
\hline \multicolumn{7}{|c|}{ Crosstab } \\
\hline & & & \multicolumn{3}{|c|}{$\begin{array}{c}\text { Years of work } \\
\text { experience in the } \\
\text { current work position }\end{array}$} & \multirow[t]{2}{*}{ Total } \\
\hline & & & $<=5$ & $6-10$ & $11+$ & \\
\hline \multirow{15}{*}{ Insomnia } & \multirow{3}{*}{ None } & Count & 36 & 37 & 35 & 108 \\
\hline & & $\%$ within insomnia & $33.3 \%$ & $34.3 \%$ & $32.4 \%$ & $100.0 \%$ \\
\hline & & $\begin{array}{l}\% \text { within years of work experience in the current } \\
\text { work position }\end{array}$ & $48.6 \%$ & $52.1 \%$ & $51.5 \%$ & $50.7 \%$ \\
\hline & \multirow{3}{*}{ Mild } & Count & 17 & 20 & 14 & 51 \\
\hline & & $\%$ within insomnia & $33.3 \%$ & $39.2 \%$ & $27.5 \%$ & $100.0 \%$ \\
\hline & & $\begin{array}{l}\% \text { within years of work experience in the current } \\
\text { work position }\end{array}$ & $23.0 \%$ & $28.2 \%$ & $20.6 \%$ & $23.9 \%$ \\
\hline & \multirow{3}{*}{ Moderate } & Count & 13 & 7 & 13 & 33 \\
\hline & & $\%$ within insomnia & $39.4 \%$ & $21.2 \%$ & $39.4 \%$ & $100.0 \%$ \\
\hline & & $\begin{array}{l}\% \text { within years of work experience in the current } \\
\text { work position }\end{array}$ & $17.6 \%$ & $9.9 \%$ & $19.1 \%$ & $15.5 \%$ \\
\hline & \multirow{3}{*}{ Severe } & Count & 5 & 4 & 5 & 14 \\
\hline & & \% within insomnia & $35.7 \%$ & $28.6 \%$ & $35.7 \%$ & $100.0 \%$ \\
\hline & & $\begin{array}{l}\% \text { within years of work experience in the current } \\
\text { work position }\end{array}$ & $6.8 \%$ & $5.6 \%$ & $7.4 \%$ & $6.6 \%$ \\
\hline & \multirow{3}{*}{ Very severe } & Count & 3 & 3 & 1 & 7 \\
\hline & & $\%$ within insomnia & $42.9 \%$ & $42.9 \%$ & $14.3 \%$ & $100.0 \%$ \\
\hline & & $\begin{array}{l}\% \text { within years of work experience in the current } \\
\text { work position }\end{array}$ & $4.1 \%$ & $4.2 \%$ & $1.5 \%$ & $3.3 \%$ \\
\hline
\end{tabular}

American Research Journal of Nursing 
Association between Insomnia, Demographic Characteristics and Self - Esteem in Nursing Personnel in Primary Care and in Emergency - Intensive Care Units on the Island of Crete

\begin{tabular}{|c|c|c|c|c|c|}
\hline \multirow{4}{*}{ Total } & Count & 74 & 71 & 68 & 213 \\
\cline { 2 - 6 } & \% within insomnia & $34.7 \%$ & $33.3 \%$ & $31.9 \%$ & $100.0 \%$ \\
\cline { 2 - 6 } & $\begin{array}{c}\text { \% within years of work experience in the current } \\
\text { work position }\end{array}$ & $100.0 \%$ & $100.0 \%$ & $100.0 \%$ & $100.0 \%$ \\
\hline
\end{tabular}

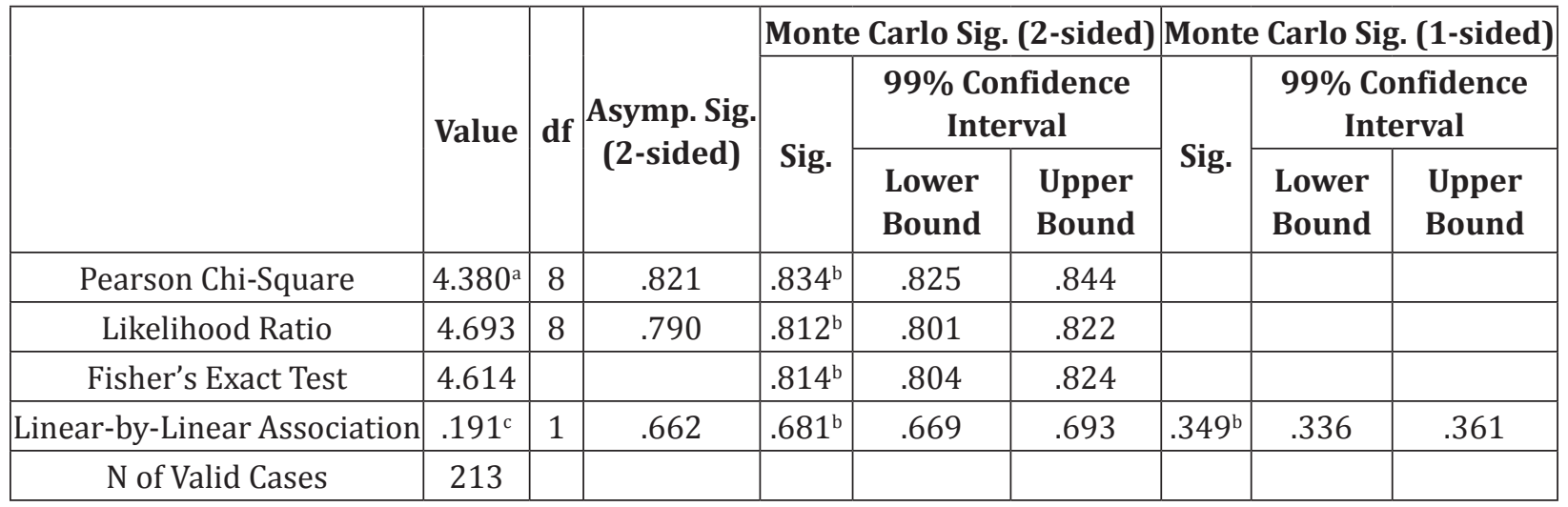

a. 6 cells $(40.0 \%)$ have expected count less than 5 . The minimum expected count is 2.23 .

b. Based on 10000 sampled tables with starting seed 423042446 .

c. The standardized statistic is -.437.

\section{Discussion}

The present research investigated the intensity symptoms of anxiety and self-esteem in a sample of 213 nursing personnel in five major hospitals and eleven Health Centers in the prefecture of Crete.

As far as the working characteristics of individuals are concerned, it was found that most nurses worked in the Intensive Care Unit (ICU) and the fewer in the Emergency Department (ED). The average length of work experience of the sample was 15.78 years and the work experience in the part that worked during the survey was 8 years. In this sample, the majority of the total sample was married $75.1 \%$ and the unmarried was $23.2 \%$. The mean age for the total sample was 41.73 years.

In the present research, the results of the Battle Questionnaire for Self-Esteem showed that $51.6 \%$ of the nursing personnel that took part in the study had a middle self-esteem, while the lowest percentage of the total sample $(8.0 \%)$ had a very high self-esteem. A study conducted in Wales, on mental health community nurses was found that most nurses had middle self-esteem [16]. Another relevant study revealed that $60.1 \%$ of nurses had an average self-esteem. The correlation of the analysis also revealed a linear negative relationship between selfesteem and emotional exhaustion. An important linear relationship was found between self-esteem and lack of personal integration [17].

The most common symptoms according Hamilton Anxiety Scale were, "stress" (33.8\%), "tension" (32.4\%), "cognitive symptoms "(26.8\%), "symptoms of muscular system"(26.3\%), "insomnia"(23.9\%), "general physical symptoms (23.5\%)," cardiorespiratory symptoms "(23.0\%)," depressed mood" (21.6\%) and" gastrointestinal symptoms" \%).

A survey conducted in 2014 showed that nursing suffered from mild intensity of anxiety symptoms, with $19.9 \%$ of nursing staff experiencing moderate intensity of anxiety symptoms and 3.9\% having a severe intensity of anxiety symptoms. Symptoms with the most frequent recordings were sleep disorders, anxiety disorder, 
Association between Insomnia, Demographic Characteristics and Self - Esteem in Nursing Personnel in Primary Care and in Emergency - Intensive Care Units on the Island of Crete

cognitive impairment, and hypertension [18]. An another survey conducted in Greece in 213 nurses who worked in the Emergency Department (GLP) of eight General Hospitals showed that the level of anxiety symptoms was moderate and the most common symptoms was sleep disorders, anxiety and depressed mood [19].

The total sample of our study experiences mild tension insomnia symptoms associated with self-esteem (p $<0.002$ ). In a study, conducted in 1,805 adults aged between 30 and 84 years in the United States and explored the relationship between sleep and self-esteem, the results showed that people with symptoms of insomnia are graded to a large extent with lower self- independent [20].

The nursing staff of the overall sample of our study shows mild insomnia symptoms associated with marital status ( $\mathrm{p}<0.026$ ). The present study showed that married women show mild to severe insomnia symptoms. Research has not been conducted to investigate the relationship between insomnia and marital status in nursing personnel. Another study in general population supported that the importance of social support has been indirectly reflected, by the higher risks of insomnia in divorced/widowed females and retired males. However, owing to the lack of detailed measurement of the social support system, further study on the relationship between insomnia and social support is indicated. In addition, different gender-specific stress-coping strategies have been suggested to explain the increased vulnerabilities in depression and insomnia in females [21].

\section{REFERENCES}

1. Jindal R, Buysse D, Thase M. Maintenance treatment of insomnia: what can we learn from the depression literature? Am J Psychiatry 2004;161:19-24.

2. Ringdahl E, Pereira S, Delzell J. Treatment of primary insomnia. J Am Board Fam Pract 2004;17:212-9.

3. Walsh, J. K. (2004). Clinical and socioeconomic correlates of insomnia. The Journal of Clinical Psychiatry, 65(Suppl8), 13-19.

4. Adriane M. Soehner, MA Allison G. Harvey. Prevalence and Functional Consequences of Severe Insomnia Symptoms in Mood and Anxiety Disorders: Results from a Nationally Representative Sample Sleep, Volume 35, Issue 10, 1 October 2012, Pages 1367-1375

5. Buysse DJ, Angst J, Gamma A, Ajdacic V, Eich D, Rössler W. Prevalence, course, and comorbidity of insomnia and depression in young adults. Sleep. 2008;31:4732.

6. Lemola S, Räikkönen K, Gomez V, Allemand M. Optimism and Self-Esteem Are Related to Sleep. Results from a Large Community-Based Sample. Int.J. Behav. Med.2013;20:567

7. AM Gregory, TC Eley, TG O'Connor, FV Rijsdijk. Family influences on the association between sleep problems and anxiety in a large sample of pre-school aged twins - Personality and Individual Differrences 39.2005;1337-1348.

8. Ohayon MM, Caulet M, Guilleminault C: Complaints about nocturnal sleep: how a general population perceives its sleep, and how this relates to the complaint of insomnia. Sleep. 1997; 20: 715-723.

9. Leger D, Guilleminault C, Dreyfus JP, Delahaye C, Paillard M: Prevalence of insomnia in a survey of 12,778 adults in France. J Sleep Res. 2000;9: 35-42. 10.1046/j.1365-2869.2000.00178.x.

10. Li, R. H. ., Wing, Y. ., Ho, S. ., \& Fong, S. Y. Gender differences in insomnia - a study in the Hong Kong Chinese population. Journal of Psychosomatic Research. 2002; 53(1), 601-609.

11. Bebbington PE. Sex and depression. Psychol Med 1998; 28(1): 1-8.

American Research Journal of Nursing

Page 15 
Association between Insomnia, Demographic Characteristics and Self - Esteem in Nursing Personnel in Primary Care and in Emergency - Intensive Care Units on the Island of Crete

12. Piccinelli M, Wilkinson G. Gender differences in depression - critical review. Br J Psychiatry 2000; 177: 486-92.

13. Roberts RE, Shema SJ, Kaplan GA, Strawbridge WJ. Sleep com-plaints and depression in an aging cohort: a prospective perspective. Am J Psychiatry 2000;157(1):81-8

14. Hamilton M. The assessment of anxiety states by rating. Br J Med Psychol 1959; 32:50-55.

15. Stalikas A, Triliva S, Rousi P (2002). Psychometric Instruments in Greece, Greek Lettering, Athens, 2002; 240-241 Author: J. Battle (Adjustment: E. Argirakouli).

16. Fothergill A, Edwards D, Hannigan B, Burnard P, Coyle D. Self-esteem in community mental health nurses: findings from the all-Wales stress study. Journal of Psychiatric and Mental Health Nursing, 2000; 7: 315-321.

17. 17. Yaghoobnia F, Mazloom SR, Ghadry JS, Esmaeili H. Study of the Relationship Between Self - Estem and Burnout in nurses of hospitals of mashad university of medicals sciences in 1379. Journal of sabzevar University of medical sciences 2003; 10:73-79.

18. Mitrousi S . Effect of anxiety on the physical and mental health of nurses. Doctoral thesis, 2014, Sparta.

19. Stathopoulou H, Karanikola MNK, Panagiotopoulou F, Papathanassoglou EDE. Anxiety levels and related symptoms in emergency nursing personnel in Greece. Journal of Emergency Nursing, 2011; 37: 314-320.

20. Lemola S, Räikkönen K, Gomez V, Allemand M. Optimism and Self-Esteem Are Related to Sleep. Results from a Large Community-Based Sample. Int.J. Behav. Med. 2013; 20:567

21. Bebbington PE. Sex and depression. Psychol Med 1998; 28(1): 1-8.

Citation: Maria Maniou, Sofia Zyga, Spyros Vliamos, Panagiotis Prezerakos, Katerina Flora, Andreas Pavlakis, "Association between Insomnia, Demographic Characteristics and Self - Esteem in Nursing Personnel in Primary Care and in Emergency - Intensive Care Units on the Island of Crete". American Research Journal of Nursing. 2019; 5(1): 1-16.

Copyright (C) 2019 Maria Maniou, Sofia Zyga, Spyros Vliamos, Panagiotis Prezerakos, Katerina Flora, Andreas Pavlakis, This is an open access article distributed under the Creative Commons Attribution License, which permits unrestricted use, distribution, and reproduction in any medium, provided the original work is properly cited.

American Research Journal of Nursing

Page 16 